Reprod. Nutr. Dévelop., 1981, 21 (1), 149-161.

\title{
Etude histologique et ultrastructurale de la gonade d'Helix aspersa Müller à l'éclosion
}

par Bernadette GRIFFOND, Jacqueline BRIDE

Laboratoire de Zoologie ef Embryologie,

L.A. CNRS no 040310,

Faculté des Sciences,

Place Leclerc, 25030 Besançon Cedex.

Summary. A sfudy of the histology and fine structure of the gonad of Helix aspersa Müller at hatching.

The gonad of young Helix aspersa, studied by light and electron microscopy during the first post-hatching week, showed a compact structure. Eventually, a lumen appeared as the celis moved apart. The gonad was composed of stem and germinal cells. The latter cells were represented by primordial germ cells and oocytes. With our method of rearing, the female germinal line differentiated first.

\section{Introduction.}

Depuis les travaux d'Ancel (1903) et de Guyard (1971), il est généralement admis que les Escargots du genre Helix sont des hermaphrodites protandres. Dans la gonade post-embryonnaire de ces animaux se différencient successivement les spermatogonies ef les cellules nourricières, puis les cellules de la lignée femelle.

A la suite de l'installation d'un élevage expérimental, nous avons réalisé une étude de l'organogenèse et de la différenciation de l'ovotestis de jeunes Helix aspersa. Nous avons ainsi pu faire des observations qui se révèlent en contradiction avec cerfaines des données antérieures. Nous nous proposons dans ce travail de présenter une description histologique et ultrastructurale de la gonade d'Helix aspersa élevés au laboratoire, en nous limitant à la première semaine qui suit l'éclosion.

\section{Matériel et méthodes.}

Matériel. - Les pontes d'Helix aspersa à partir desquelles a été étudiée la gonade post-natale proviennent d'animaux élevés au laboratoire dans des conditions contrôlées de température $\left(20^{\circ}\right)$, humidité (saturation), alimentation (commercialisée par Sanders ( $\left.{ }^{1}\right)$ ). Les pontes sont conservées à l'obscurité, dans les mêmes conditions

(1) 17, quai de l'Industrie, 91200 Athis-Mons. 
de température et d'humidité. L'âge des parents est connu de même que la date de ponte et le jour de naissance des jeunes. Notre étude porte sur 32 Escargots âgés de 1 à 7 jours, prélevés dans 6 pontes différentes et fixés en juin 1977, octobre 1978, octobre 1979 et février-mars 1980.

Techniques. - Après élimination de la coquille, les tortillons ont été fixés pendant $2 \mathrm{~h}$ dans une solution de glutaraldéhyde à 2 p. 100 dans un tampon cacodylate de sodium $0,1 \mathrm{M}(P O=250 \mathrm{mOsm})$. Après un lavage de 12 à $16 \mathrm{~h}$ dans le tampon cacodylate $0,1 \mathrm{M}$ additionné de $\mathrm{NaCl} 0,16 \mathrm{M}(\mathrm{PO}=250 \mathrm{mOsm})$, ils ont subi une postfixation d'1 h à l'acide osmique à 2 p. 100 dans le tampon cacodylate $0,2 \mathrm{M}$ $(P O=240 \mathrm{mOsm})$. Après déshydratation selon la méthode de Luft (1961), les tortillons ont été inclus dans le mélange épon-araldite (Anderson et Ellis, 1965) dans lequel la proportion de DMP 30 a été ramenée à 1,7 p. 100 suivant la méthode de Mollenhaver (1964). Les coupes semi-fines ont été colorées au bleu de toluidine (Trump ef al., 1961), les coupes ultra-fines contrastées à l'acétate d'uranyle puis au citrate de plomb (Reynolds, 1963).

\section{Résultats.}

\section{Morphologie de la gonade.}

Chez des Escargots fixés le jour de la naissance, la gonade occupe une position superficielle, à la face interne de l'extrémité du tortillon. Elle est localisée dans le tissu conjonctif qui sépare la poche nourricière (poche de résorption de l'albumen) et la paroi du corps. Elle se présente sous l'aspect d'un cordon plein, plus ou moins dilaté en massue, mesurant entre 80 ef $100 \mu \mathrm{m}$ de longueur sur 15 à $20 \mu \mathrm{m}$ de diamètre. Durant la première semaine post-natale, elle s'enfonce progressivement entre les replis de la poche nourricière (fig. 1, PI. I) tandis que s'organise autour d'elle un tissu conjonctif particulier ou tissu vésiculeux (fig. 2, PI. I).

D'une ponte à l'autre et entre individus d'une même ponte, de grandes variations sont observées tant en ce qui concerne la localisation gonadique qu'en ce qui concerne le creusement d'une lumière. Au moment de la naissance, le canal hermaphrodite, tapissé d'un épithélium cubique unistratifié et creusé d'une large lumière, est en communication avec l'extrémité antérieure du massif gonadique. Vers le 5 e jour apparaît dans ce dernier une fente très étroite, en continuité avec la lumière du canal hermaphrodite (fig. 2, Pl. I). Cette fente s'élargit par écartement des cellules et gagne de proche en proche la partie postérieure de la gonade.

\section{Etude histologique de la gonade.}

Dès la naissance, l'observation en microscopie photonique de la gonade des jeunes Helix aspersa permet de repérer des cellules germinales et des cellules-souches. Les cellules-souches présentent un noyau petit et sombre, à chromatine en mottes ; leur cytoplasme renferme souvent de grandes plages faiblement colorables au bleu de toluidine (fig. 1 et $2, \mathrm{PI}$. I). 
Les cellules germinales contrastent fortement avec les précédentes par l'aspect clair de leur cytoplasme et de leur noyau. Leur nombre, généralement faible au moment de la naissance (4 à 6), varie d'un individu à l'autre ef s'accroît rapidement. Durant la première semaine post-natale, 2 catégories de cellules germinales sont particulièrement bien représentées. Ce sont :

- d'une part des cellules que nous appellerons cellules germinales primordiales. Piriformes ou ovales, elles sont toujours allongées parallèlement au bord de la gonade. Elles possèdent un noyau clair dans lequel sont visibles 2 ou 3 nucléoles denses (fig. 1 . PI. I) ;

- d'autre part des ovocytes : dans 18 gonades sur 32 prélevées chez des animaux âgés de 1 à 7 jours et issus de 6 pontes différentes, nous avons pu identifier avec certitude des ovocytes en auxocytose, généralement au nombre d'1 ou 2 par gonade (fig. 3, PI. I).

A côté de ces deux types cellulaires, des cellules germinales orientées de façon variable par rapport à la paroi de la gonade sont parfois observées, dans les gonades les plus évoluées. Ces cellules feront l'objet de recherches ultérieures. Enfin dans 2 seulement des 32 gonades âgées de 1 à 7 jours, de rares spermatogonies (1 ou 2) sont présentes.

\section{Efude ultrastructurale des cellules de la gonade post-embryonnaire.}

a) Les cellules-souches. - Ce sont les cellules les mieux représentées dans la gonade post-natale. Elles peuvent être disposées irrégulièrement, ou organisées en un épithélium. Elles sont caractérisées par un noyau à contour plus ou moins lobé dans lequel des mottes irrégulières d'hétérochromatine sont dispersées au sein du nucléoplasme (fig. 4, PI. I). Un nucléole souvent entouré de chromatine associée occupe une position subcentrale. Le cytoplasme abondant est en grande partie occupé par une ou plusieurs vastes plages renfermant de fines particules ou des rosettes de glycogène. Ces zones de réserves occupent des positions basale et apicale dans les cellules organisées en épithélium. En dehors des ribosomes très abondants, qui confèrent au cyłoplasme un aspect extrêmement dense, les organites cellulaires sont assez peu nombreux : ergastoplasme représenté par quelques citernes aplaties, mitochondries à matrice sombre, dictyosomes très rares. Dans la région centrale de la gonade, lorsque la lumière n'est encore que virtuelle, puis dans la zone apicale des cellules, des complexes jonctionnels unissent entre elles les cellules-souches voisines; ils sont composés de jonctions septées plus ou moins étendues et d'un desmosome apical (fig. 5, PI. I). L'apex des cellules-souches, souvent riche en microlubules, est hérissé de quelques villosités tandis que leur partie basale émet des prolongements pseudopodiaux qui s'étirent le long de la lame basale où ils constituent des enchevêtrements compliqués (fig. 6, Pl. 1). De nombreuses divisions de cellules-souches peuvent être observées dans la gonade post-embryonnaire et des ponts intercellulaires persistent fréquemment entre les cellules-filles.

L'utilisation des réserves se traduit souvent par la présence au niveau des plages polysaccharidiques de zones transparentes aux électrons ou de figures membranaires plus ou moins développées. 
Dans la zone de transition entre la gonade et le canal hermaphrodite (fig. 1, PI. II), les cellules constituent dès la naissance un épithélium unistratifié. Ce sont des cellules toutes semblables, à noyaux irréguliers renfermant de nombreuses mottes de chromatine et à cytoplasme occupé dans sa presque totalité par des amas de substance polysaccharidique. Elles ne se distinguent guère des cellules-souches gonadiques que par leur organisation épithéliale et la plus grande abondance de leurs réserves. Parmi ces cellules, quelques cellules germinales primordiales sont disséminées (fig. 1 , PI. II).

b) Les cellules germinales primordiales. - Ce sont de grandes cellules piriformes ou ovales, mesurant $20 \mu \mathrm{m} \times 7 \mu \mathrm{m}$, toujours proches du bord de la gonade. Elles sont allongées parallèlement à la lame basale (fig. 1 et 2 , PI. II) dont elles sont séparées par les prolongements des cellules-souches mais avec laquelle elles conservent cependant toujours une étroite zone de contact (fig. 3, PI. II). Elles sont isolées de la lumière, lorsque celle-ci est creusée, par au moins une assise de cellules-souches (fig. 2, PI. II). Leur noyau ovoïde, très clair, renferme quelques petits amas d'hétérochromatine souvent disposés en fines travées. Dans les nucléoles (1 à 3), à contour très irrégulier (fig. 1 et 4, PI. II), substance fibrillaire et substance granulaire apparaissent intimement mêlées.

Dans le cytoplasme, les polyribosomes sont nombreux. L'ergastoplasme constitue une longue citerne aplatie à la périphérie du noyau. Les mitochondries possèdent des crêtes transversales tandis que plusieurs dictyosomes sont groupés au pôle le plus

\section{PLANCHE I}

FIG. 1. - Gonade d'un Escargof âgé de 4 jours. C'est un massif cellulaire compact dans lequel on reconnaît une cellule germinale primordiale (cgp) $(\times 480)$.

cs : cellule-souche; $G$ : gonade; $H$ : hépatopancréas ; tv : tissu vésiculeux.

FIG. 2. - Coupe longitudinale de la gonade (G) et du canal hermaphrodite (ch) d'un Escargot de 5 jours. La lumière gonadique se met en place dans le prolongement de la lumière du canal par écartement des cellules $(\times 480)$.

FIG. 3. - Coupe oblique de la gonade (G) et du canal hermaphrodife (ch) d'un Escargot de 6 jours. 2 ovocytes en prévitellogenèse (ov) sont visibles $(\times 480)$.

FIG. 4. - Aspect d'une cellule-souche (cs) dans la gonade d'un animal de 2 jours. La cellule s'étend de la lame basale (Ib) à la lumière de la gonade (L). Le noyau ( $N$ ) occupe une position basale. Le cytoplasme renferme de grandes plages glycogéniques $(\mathrm{g} l)$. Des complexes jonctionnels (j) sont visibles dans la région apicale $(\times 11500)$. $m$ : mitochondrie.

FIG. 5. - Complexe jonctionnel unissant entre elles des cellules-souches (cs) : il est constitué par la succession d'une zone de jonctions septées (js) ef d'un desmosome apical (da) $(\times 30000)$. L : lumière.

FIG. 6. - Les pseudopodes émis par les cellules-souches constifuent à proximité de la lame basale (Ib) des enchevêtrements compliqués $(\times 11500)$. 
large de la cellule. De rares inclusions denses ef homogènes sont visibles ainsi que des corps multivésiculaires mais les cellules germinales primordiales ne renferment pas ou peu de réserves polysaccharidiques.

\section{Relations entre la gonade et les tissus voisins.}

Durant les premiers jours qui suivent la naissance, la lame basale qui limite la gonade est extrêmement ténue $(20 \mathrm{~nm})$ et souvent discontinue. Même dans une gonade renfermant déjà des ovocytes bien différenciés, les limites peuvent être encore imprécises et mal définies (PI. III et fig. 1, Pl. IV). Quelques rares filaments de collagène, fins et inorganisés, sont visibles dans le tissu conjonctif qui limite l'ébauche gonadique, du côté de la poche nourricière. A ce niveau, les cellules les plus externes (PI. III et fig. 1, PI. IV) du massif gonadique possèdent des caractères particuliers (présence d'un réseau ergastoplasmique dilaté, abondamment garni de ribosomes et renfermant une substance moyennement opaque aux électrons), similaires aux caractères des cellules observées dans le tissu conjonctif environnant. Des contacts s'établissent entre les cellules gonadiques périphériques et des cellules conjonctives (fig. 1, PI. IV). Il semble donc bien qu'à ce stade, la gonade s'accroisse non seulement par multiplication cellulaire mais également par intégration de cellules conjonctives qui perdent ensuite leurs caractères propres. Dans cette même région, il n'est pas rare que les cellules-souches émettent de très longs pseudopodes $(10$ à $15 \mu \mathrm{m})$ qui s'enfoncent dans le tissu conjonctif (fig. 2, PI. IV) et sont soutenus par de véritables faisceaux de microtubules. Enfin des contacts directs sont également observés entre la gonade et des prolongements nerveux, aucune lame basale ne s'interposant entre les deux tissus (fig. 3, PI. IV).

\section{PLANCHE II}

FIG. 1. - Coupe oblique au niveau du canal hermaphrodite (ch) d'un Escargot de 7 jours. Les cellules de type épithélial ont un noyau riche en hétérochromatine ef un cytoplasme encombré de glycogène (gl). Une cellule germinale primordiale (cgp) est reconnaissable à son noyau clair et à son cytoplasme dépourvu de réserves $(\times 4500)$.

FIG. 2. - Cellule germinale primordiale (cgp) observée dans la gonade d'un Escargot de 7 jours. Elle a une allure piriforme et un noyau $(\mathrm{N})$ clair. Les mitochondries $(\mathrm{m})$ sont abondantes ef l'appareil de Golgi $(\mathrm{G})$ bien représenté $(\times 8400)$.

FIG. 3. - Du côté de la paroi gonadique, la cellule germinale primordiale (cgp) a un contact étroit avec la lame basale (lb) ( $\times 32400)$. $m$ : mitochondrie ; mt : microtubule.

FIG. 4. - Le nucléole des cellules germinales primordiales, fibrillogranulaire, présente des taches claires, d'aspect identique à celui du nucléoplasme $(\times 26900)$. 


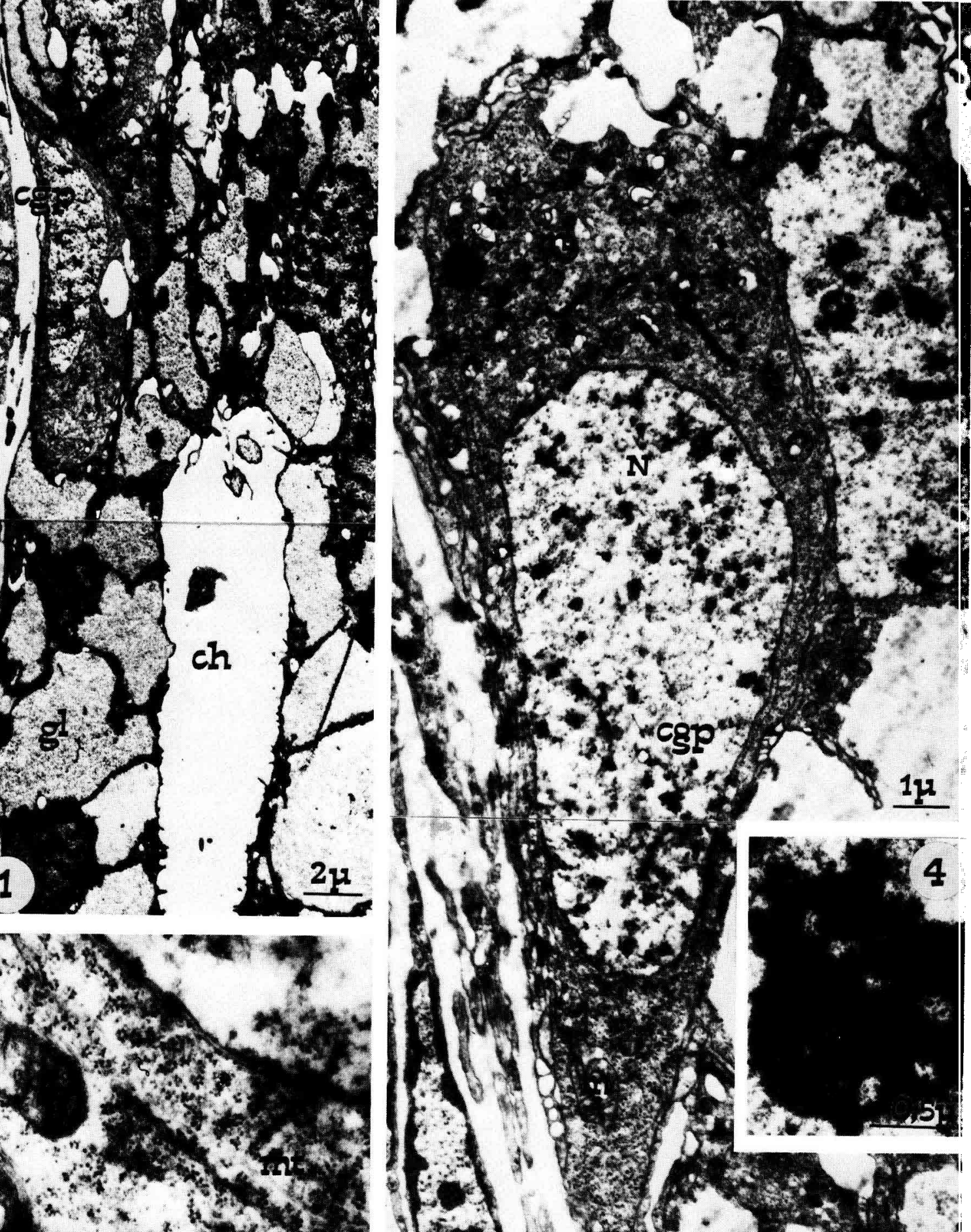

(1) 10

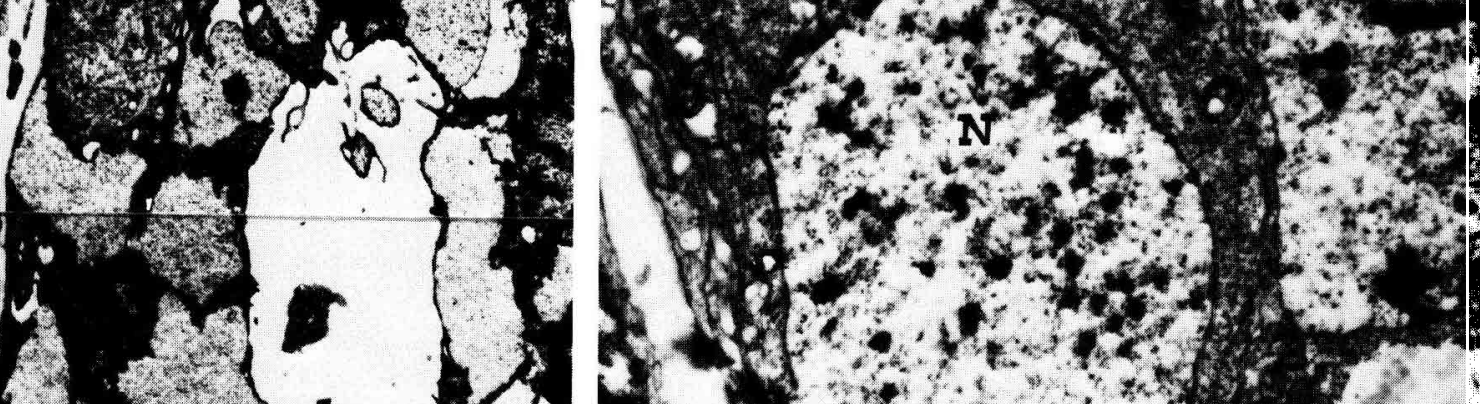
(Whitis

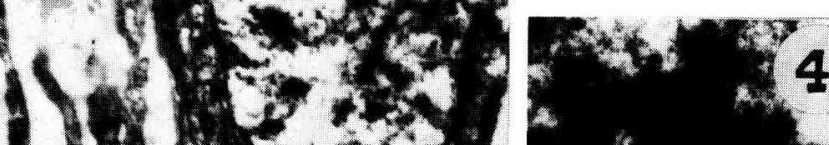

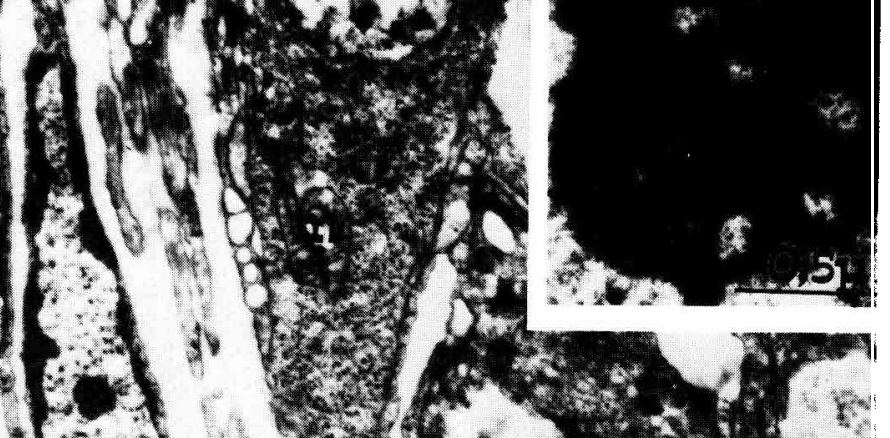

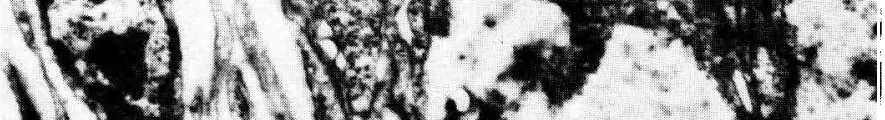

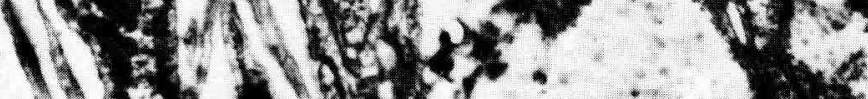

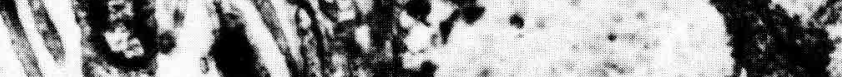
1. \& $\left.\mathrm{F}^{2}, \mathrm{k}, 3^{2}\right)$

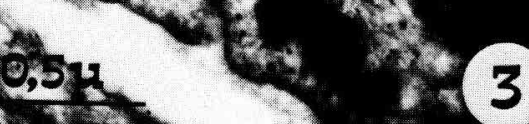

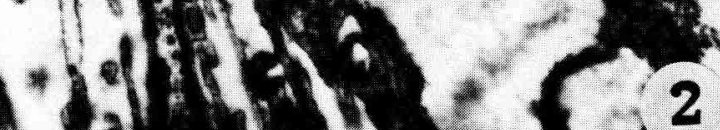

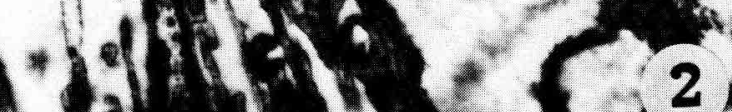




\section{Discussion.}

1) Origine de la gonade. - Chez les Pulmonés, les avis sont très partagés quant à l'origine de la gonade. En effet si chez les Basommatophores, il est admis que la gonade a une origine mésodermique (Fraser, 1946 ; Brisson et Regondaud, 1971, 1977), chez certains Stylommatophores, au contraire, elle dériverait de l'ectoderme (Hoffman, 1922 ; Laviolette, 1954 ; Luchtel, 1972) ; chez l'Escargot en particulier, les travaux antérieurs aboutissent à des points de vue opposés : pour Rouzaud (1885), la gonade est issue de l'ectoderme tandis que pour Von Jehring (1875), Ancel (1903), Buresch (1912), elle esł d'origine mésodermique. Nos observations nous permettent actuellement d'affirmer que chez Helix aspersa, des cellules mésodermiques sont incorporées à la gonade, durant les jours qui suivent la naissance. Une étude de la gonade embryonnaire devrait fournir des renseignements relatifs aux premières étapes de mise en place de la gonade.

2) Chronologie de la différenciation des cellules germinales. - En ce qui concerne la différenciation des lignées mâle et femelle, Ancel (1903), Gatenby (1917) et Guyard (1971, 1976) observent d'abord la différenciation des cellules mâles puis celle des cellules nourricières et enfin, lorsque la lumière des tubules est comblée par la multiplication spermatogoniale, celle des cellules femelles. Pour les deux premiers auteurs, les cellules germinales se différencient par simple évolution des cellules-souches qui passent par un stade « cellule progerminative indifférente » avant de s'orienter vers le sens mâle ou vers le sens femelle.

Pour Guyard (1971), certaines cellules-souches après avoir subi un accroissement du noyau (protogonies) se divisent de façon inégale pour donner une cellule nourricière qui demeure pariétale et une spermatogonie primordiale qui sort de l'épithélium germinatif ; après remplissage des tubules par les cellules mâles, les « protogonies» qui persistent dans l'épithélium germinatif évoluent in situ en cellules femelles; les cellules-souches voisines s'organisent alors en un follicule.

Dans les coupes que nous avons observées, diverses images, en particulier l'existence de ponts entre cellules-souches ef cellules germinales, suggèrent que la première génération de cellules germinales se différencie par division inégale de cellulessouches. Ce processus serait valable pour la lignée mâle comme le pensait Guyard (1971) mais également pour la lignée femelle car nous avons pu reconnaître la persistance d'une continuité cytoplasmique entre des ovocytes et l'une des cellules qui constituent le follicule (fig. 4, PI. IV).

\section{PLANCHE III}

\section{Coupe oblique dons la gonade d'un Escargot à l'éclosion}

La lame basale (lb) est très mince et par endroits inexistante. A l'extérieur de cette lame basale, des cellules conjonctives (cc), d̀ noyau allongé, sont plaquées contre la gonade. Dans celle-ci, on peut reconnaître des cellules-souches (cs) ainsi que deux ovocytes (ov) en prévitellogenèse. La lumière n'est encore que virtuelle $(\times 4000)$. gl : glycogène ; j : complexe jonctionnel ; tn : tissu nerveux. 
En ce qui concerne les cellules germinales de première génération apparues le long de la lame basale, leur devenir n'est pas clair. Leur localisation et leur forme tendraient à les faire assimiler à des ovogonies primordiales qui se différencient en ovocytes soit directement, soit à la suite de mitoses. Cependant aucun critère ne permet d'écarter l'hypothèse de cellules germinales primordiales indifférenciées qui, en fonction du contexte physiologique, donneront naissance à la lignée mâle ou à la lignée femelle. Quoi qu'il en soit, les premiers ovocytes sont présents avant que des cellules mâles puissent être reconnues.

Nos résultats relatifs à l'évolution chronologique de la différenciation des types cellulaires dans la gonade d'Helix aspersa sont comparés à ceux de Guyard (1971) dans le tableau 1.

\section{TABLEAU 1}

Types cellulaires présents dans la gonade post-natale d'Helix aspersa.

\begin{tabular}{|c|c|c|c|}
\hline Age de la gonade & d'après Guyard & d'après Griffond et Bride & $\begin{array}{c}\text { Age de } \\
\text { la gonade }\end{array}$ \\
\hline Naissance à 9 jours & Cellules-souches & \multirow{2}{*}{$\begin{array}{l}\text { Cellules-souches } \\
\text { Cellules germinales } \\
\text { primordiales } \\
\text { Ovocytes } \\
\text { Cellules folliculeuses }\end{array}$} & \multirow[b]{2}{*}{$\begin{array}{c}\text { Naissance } \\
\text { à } \\
10 \text { jours }\end{array}$} \\
\hline 10 jours & $\begin{array}{l}\text { Cellules-souches } \\
\text { Profogonies = cellules- } \\
\text { souches à noyau dilaté }\end{array}$ & & \\
\hline Après le $10^{\mathrm{e}}$ jour & $\begin{array}{c}\text { Cellules-souches } \\
\text { Protogonies } \\
\text { Spermatogonies } \\
\text { Cellules nourricières }\end{array}$ & \multirow{2}{*}{$\begin{array}{l}\text { Cellules-souches } \\
\text { Cellules germinales } \\
\text { primordiales } \\
\text { Ovocytes } \\
\text { Cellules folliculeuses } \\
\text { Spermatogonies } \\
\text { Cellules nourricières }\end{array}$} & \multirow[b]{2}{*}{$\begin{array}{l}\text { Après le } \\
10^{\circ} \text { jour }\end{array}$} \\
\hline $\begin{array}{l}\text { Après que la multipli- } \\
\text { cation spermatogo- } \\
\text { niale ait comblé la } \\
\text { lumière des tubules }\end{array}$ & $\begin{array}{c}\text { Cellules-souches } \\
\text { Protogonies } \\
\text { Spermatogonies } \\
\text { Cellules nourricières } \\
\text { Ovocytes } \\
\text { Cellules folliculeuses }\end{array}$ & & \\
\hline
\end{tabular}

\section{PLANCHE IV}

FIG. 1. - Un plus fort grossissement de la planche III montre qu'à l'éclosion, la lame basale n'est pas continue autour de la gonade. Des cellules à caractères conjonctifs (cc) s'accolent au massif gonadique. Elles sont caractérisées par un ergastoplasme (er) très riche en ribosomes et fortement dilaté. L'enveloppe nucléaire est elle aussi abondamment garnie de ribosomes (flèches) ( $\times 11$ 400).

FIG. 2. - Aspect d'une cellule-souche (cs) dans une gonade fixée le jour de l'éclosion : elle a un contour irrégulier et émet de longs pseudopodes ( $\times 8900$ ).

FIG. 3. - Des contacts directs s'établissent entre les cellules-souches (cs) et une ramification nerveuse (tn) avant la mise en place de la lame basale, dans une gonade fixée le jour de l'éclosion $(\times 19000)$. $\mathrm{gl}$ : glycogène ; $m$ : mitochondrie; $N$ : noyau.

FIG. 4. - Dans une gonade juvénile, une continuité cytoplasmique est visible (flèche) entre un ovocyte (ov) ef une cellule folliculeuse (cf.) La lame basale (lb) est épaisse (X 22900), mt : microlubule. 
3) Signification de la différenciation précoce des ovocyfes. - La présence d'ovocytes dans la gonade post-embryonnaire d'Helix aspersa, en absence de cellules de la lignée mâle, s'explique aisément par la théorie de l'autodifférenciation ovocytaire (Charniaux-Cotton, 1957) : chez nos animaux, dans les jours qui suivent la naissance, les facteurs d'origine cérébrale, nécessaires à la manifestation de la lignée mâle (Guyard, 1971 ; Gomot, 1973) sont encore absents ou présents en faible quantité, laissant s'exprimer la tendance spontanée à l'autodifférenciation femelle. Nos résultats sont à rapprocher de ceux obtenus chez Litforina littorea, Prosobranche à sexes séparés chez lequel les auteurs signalent une orientation femelle primitive de la gonade des jeunes mâles (Streiff et al., 1979 ; Herlin, 1980).

Cette différenciation ovocyłaire précoce traduit donc, par rapport aux observations de Guyard (1971) réalisées sur un matériel prélevé dans des conditions différentes, des modifications dans les processus de différenciation des lignées germinales. Plusieurs hypothèses peuvent être proposées pour tenter d'expliquer les variations observées dans la chronologie d'apparition des divers types cellulaires de la gonade. Les divergences pourraient être liées à la constitution génétique des parents, ou bien à la période de ponte ou plus vraisemblablement aux méthodes d'élevage utilisées. En effet, l'influence des facteurs externes sur le fonctionnement de la gonade adulte est connue chez les Pulmonés. Les conditions de température (Bank, 1931 ; Bouillon, 1956 ; Lusis, 1966 ; Parivar, 1978), le degré d'humidité (Arias et Crowell, 1963 ; Parivar, 1978) ou la durée d'éclairement (Henderson et Pelluet, 1960 ; Sokolove et McCrone, 1978 ; McCrone et Sokolove, 1979) favorisent l'une ou l'autre des lignées germinales. Il n'est pas impossible que ces mêmes facteurs écophysiologiques aient une importance prépondérante, directe ou indirecte, au niveau de la sexualisation des cellules germinales.

En définitive, le fait que les cellules germinales mâles ou les cellules germinales femelles apparaissent en premier lieu dans la gonade d'Helix aspersa démontre l'inanité chez cette espèce d'une opposition fondamentale entre différenciation protandre et différenciation protogyne.

Reçu en août 1980.

Accepté en octobre 1980.

\section{Références}

ANCEL P., 1903. Histogenèse et structure de la glande hermaphrodite d'Helix pomatia. Arch. Biol., 19, 389-652.

ANDERSON W. A., ELLIS R. A., 1965. Ultrastructure of Trypanosoma lewisi : flagellum, microtubules and the kinetoplast. J. Protozool., 12, 483-499.

ARIAS R. O., CROWELL H. L., 1963. A contribution to the biology of the grey garden slug. Bull. Calif. Acad. Sci., 62, 83-97.

BANK O., 1931. Der Einfluss hoher Temperatur auf die Gonade von Helix pomatia. Biologia gen., 7, 429-444.

BOUILLON J., 1956. Influence of temperature on the histological evolution of the ovotestis of Cepaea nemoralis L. Nature, 117, 142-143.

BRISSON P., REGONDAUD J., 1971. Observations relatives à l'origine dualiste de l'appareil génifal chez quelques Gastéropodes Pulmonés Basommatophores. C. R. Acad. Sci. Paris, sér. D, 273, 2339-2341. 
BRISSON P., REGONDAUD J., 1977. Origine et structure de l'ébauche de la gonade chez les Gastéropodes Pulmonés Basommatophores. Malocologia, 16, 457-466.

BURESCH I., 1912. Untersuchungen über die Zwitterdrüse der Pulmonaten. I. Die Differenzierung der Keimzellen bei Helix arbustorum. Arch. Zellforsch., 7, 314-343.

CHARNIAUX-COTTON H., 1957. Croissance, régénération et déterminisme endocrinien des caractères sexuels d'Orchestia gammarella Pallas, Crustacé Amphipode. Ann. Sci. nat. Zool. Biol. anim., 19, 411-559.

FRASER L. A., 1946. The embryology of the reproductive tract of Limnoea stagnalis appressa Say. Trans. amer. micr. Soc., 65, 279-298.

GATENBY J. B., 1917. The cytoplasmic inclusions of the germ-cells. II. Helix aspersa. Quart. J. micr. Sci., 62, 555-611.

GOMOT L., 1973. Efude du fonctionnement de l'appareil génital de l'Escargot Helix aspersa par la méthode des cultures d'organes. Arch. Anat. Hist. Embr. norm. exp., 56, 131-160.

GUYARD A., 1971. Etude de la différenciation de l'ovotestis ef des facteurs contrôlant l'orientation sexuelle des gonocytes de l'Escargot Helix aspersa Müller. Th. Doct. Sci. Nat., Besançon, $\mathrm{n}^{\circ} 56$.

GUYARD A., 1976. Aspects du centrosome et des autres organites cellulaires au cours de l'orientation sexuelle des gonocytes au sein de la gonade post-embryonnaire du Mollusque Gastéropode hermaphrodite Helix aspersa Müller. Haliotis, 6, 297-313.

HENDERSON N. E., PELLUET D., 1960. The effect of visible light on the ovotestis of the slug Deroceras reticulatum (Müller). Canad. J. Zool., 38, 173-178.

HERLIN D., 1980. Contribution à l'étude de l'organogenèse post-larvaire de l'appareil génital mâle chez un Gastéropode Prosobranche Gonochorique : Liftorina littorea (L.). Thèse $3 \mathrm{e}$ cycle, Caen.

HOFFMAN H., 1922. Ueber die Entwicklung der Geschlechtsorgane bei Limax maximus. Z. Wiss. Zool., 119, 493-536.

LAVIOLETTE P., 1954. Etude cytologique et expérimentale de la régénération germinale après castration chez Arion rufus L. Ann. Sci. nat. Zool., 16, 427-535.

LUCHTEL D., 1972. Gonadal development and sex determination in pulmonate molluscs. I. Arion circumscriptus. Z. Zellforsch., 130, 279-301.

LUFT J. H., 1961. Improvements in epoxy resin embedding methods. J. biophys. biochem. Cytol., 9, $409-414$.

LUSIS O., 1966. Changes induced in the reproductive system of Arion rufus afer $L$. by varying environmental conditions. Proc. malac. Soc. London, 37, 19-26.

MCCRONE E. J., SOKOLOVE P. G., 1979. Brain-gonad axis and photoperiodically-stimulated sexual maturation in the slug, Limax maximus. J. comp. Physiol., 133, 117-124.

MOLLENHAUER H. H., 1964. Plastic embedding mixtures for use in electron microscopy. Stain Technol., 39, 111-114.

PARIVAR K., 1978. A histological survey of gonadal development in Arion ater L. (Mollusca, Pulmonata). J. moll. Stud., 44, 250-264.

REYNOLDS E. S., 1963. The use lead citrate at high $\mathrm{pH}$ as an electron opaque stain in electron microscopy. J. Cell Biol., 17, 208-212.

ROUZAUD H., 1885. Recherches sur le développement des organes géniłaux de quelques Gastéropodes hermaphrodites. Travaux Lab. Zool. Fac. Sci. Montpellier., 1, 1-133.

SOKOLOVE P. G., McCRONE E. J., 1978. Reproductive maturation in the slug, Limax maximus, and the effects of artificial photoperiod. J. comp. Physiol., 125, 317-325.

STREIFF W., LE BRETON J., HERLIN D., 1979. Gonocytes el différenciation sexuelle chez un Mollusque Gastéropode Prosobranche gonochorique Littorina littorea L. Ann. Soc. fr. Biol. Dév., Réseau Cellules germinales, 19-20.

TRUMP B. F., SMUCKLER E. A., BENDITT E. P., 1961. A method for staining epoxy sections for light microscopy. J. Ultrastruct. Res., 5, 343-345.

VON JEHRING H., 1875. Ueber die Entwicklungsgeschichte von Helix. lena Z. Naturwiss., 9, 299-336. 